\title{
Loratadine Alleviates Advanced Glycation End Product-Induced Activation of NLRP3 Inflammasome in Human Chondrocytes
}

This article was published in the following Dove Press journal:

Drug Design, Development and Therapy

\author{
Feng Gao' \\ Shanyong Zhang ${ }^{2}$ \\ 'Department of Orthopedics, The \\ Second Hospital of jilin University, \\ Changchun City, Jilin Province 13004I, \\ People's Republic of China; ${ }^{2}$ Department \\ of Spine Surgery, The Second Hospital of \\ jilin University, Changchun City, Jilin \\ Province I3004I, People's Republic of \\ China
}

Background: Chondrocytes in joint tissue are responsible for the synthesis and degradation of the cartilage matrix. Chondrocytes have been closely linked to the pathogenesis of osteoarthritis and cartilage damage. Targeted drug intervention directed at chondrocyte function is a promising strategy for the treatment of osteoarthritis. The effects of histamine receptor $\mathrm{H} 1 \mathrm{H}$ ( R) and its antagonist loratadine in osteoarthritic chondrocytes are less known.

Materials and Methods: The inhibitory effects of loratadine on NLRP3 inflammasome and the NADPH oxidase subunit NOX4 were assessed in advanced glycation end products (AGEs)-treated SW1353 chondrocytes by real-time PCR, ELISA, and Western blot experiments. The mitochondrial ROS level was measured using the specific probe MitoSOX Red. The dependent effect of loratadine on the transcriptional factor nuclear factor erythroid 2-related factor 2 (NRF2) was evaluated through an oligo-based siRNA knockdown approach and Western blot analysis.

Results: The expression of H1R was dose-responsively induced by AGEs in chondrocytes. Treatment with loratadine mitigated AGEs-induced oxidative stress, as revealed by suppressed production of mitochondrial ROS and the NADPH oxidase subunit NOX4. Loratadine treatment inhibited the expression of TxNIP and several components of the NLRP3 inflammasome complex, including NLRP3, ASC, and cleaved caspase 1 (P10). Moreover, loratadine suppressed the expression of NRF2, and the silencing of NRF2 abolished the suppressive effect of loratadine on NLRP3 inflammasome activation.

Conclusion: Our study demonstrates that loratadine protects chondrocytes from AGEsinduced TxNIP/NLRP3 inflammasome activation by modulating the expression of the transcriptional factor NRF2. This finding implies that loratadine has therapeutic potential in the treatment of osteoarthritis and cartilage injury.

Keywords: histamine H1 receptor, loratadine, NLRP3 inflammasome, NRF2, chondrocyte

\section{Introduction}

Chondrocytes in cartilage connective tissue comprise a unique cell population as they can both produce and degrade the cartilage matrix. In healthy conditions, chondrocytes are responsible for maintaining a balance between the synthesis of new extracellular matrix and the removal of old cartilage tissues. Chondrocytes are highly sensitive cells, and inflammation due to mechanical injury of the joint or connective tissues often provokes their activation and reduces the residential chondrocyte population. This leads to an imbalance in the regulatory actions of
Correspondence: Shanyong Zhang Department of Spine Surgery, The Second Hospital of Jilin University, No. $218, \mathrm{Zi}$ Qiang Street, Changchun City, Jilin Province I3004I, People's Republic of China

Tel $+86-43$ I-8II 36890

Fax $+86-431-81136993$

Email shanyong221@163.com 
chondrocytes, thereby inducing irreversible cartilage damage. Chondrocyte dysregulation has been linked with different joint diseases, such as degenerative osteoarthritis and cartilage injury. ${ }^{1}$ Stress or inflammation-induced activation of chondrocytes causes abnormal phenotypic changes and triggers the production of pro-inflammatory mediators and matrix metalloproteinases, which are harmful to cartilage. ${ }^{2}$ Advanced glycation end products (AGEs) are glycated compounds generated from the reaction between reducing sugars and amine residues on proteins or lipids. The accumulation of AGEs in the body is a major risk factor for degenerative diseases and aging. ${ }^{3}$ Previous investigations have shown that the accumulation of AGEs in articular cartilage is an important source of chondrocyte activation and cartilage damage. ${ }^{4}$ Recent progress shows that AGE-induced inflammation involves the activation of the NLRP3 inflammasome complex, suggesting that the NLRP3 inflammasome is an important mechanism in tissue injury and repair. ${ }^{5}$ Activation of the NLRP3 inflammasome leads to caspase 1-dependent release of the pro-inflammatory cytokines interleukin (IL)-1 $\beta$ and IL-18. The formation of the NLRP3 inflammasome complex is regulated by several key factors, including the anti-inflammatory factor nuclear factor erythroid 2-related factor 2 (NRF2), which negatively regulates inflammasome formation. ${ }^{6} \mathrm{NRF} 2$ functions as an anti-oxidative regulator by controlling the production of antioxidant proteins and regulating the function of the NLRP3 inflammasome, thereby contributing to its function in oxidative signaling. ${ }^{7}$

Histamine receptor 1 (H1R), a type of G protein-coupled receptor, is an important member of the histamine receptor family. ${ }^{8}$ Previous research has shown that H1R is expressed in human chondrocytes and is responsive to histamine stimulation, which induces the production of numerous proinflammatory mediators and several matrix metalloproteinases (MMPs). ${ }^{9-11}$ Additionally, H1R activation has been shown to promote proteoglycan synthesis in chondrocytes. ${ }^{12}$ These facts indicate that H1R signaling could play a role in chondrocyte regulation. H1R antagonists have been used to relieve allergenic symptoms for decades and provide a powerful tool to study the function of the $\mathrm{H} 1$ receptor. Loratadine is a second-generation antihistamine, which acts as an H1R blocker. ${ }^{13}$ Loratadine has been widely used to treat allergic symptoms associated with hay fever, seasonal allergies, and atopic dermatitis. ${ }^{14}$ In addition, loratadine has displayed pleiotropic functions in different types of cells and tissues. $^{15-17}$ However, the roles of loratadine in the formation and activation of the NLRP3 inflammasome are still unknown. In this study, we investigated the beneficial effects of loratadine against AGE-induced cytotoxicity in human SW1353 chondrocytes.

\section{Materials and Methods Cell Culture and Treatment}

The human chondrosarcoma cell line SW1353 cells used in our study were purchased from ATCC. The cells were routinely maintained in Leibovitz's L-15 medium supplemented with $10 \%$ FBS and $1 \%$ compound antibiotics (100 units $/ \mathrm{mL}$ penicillin, $100 \mu \mathrm{g} / \mathrm{mL}$ streptomycin). All cell cultures were kept in a humidified incubator environment with 95\% air and $5 \% \mathrm{CO}_{2}$ at $37^{\circ} \mathrm{C}$. The AGE cell treatment reagent was freshly prepared in $150 \mathrm{mg} / \mathrm{mL}$ stock solution. Loratadine was obtained from Sigma-Aldrich, USA. For the treatment experiments, SW1353 cells were plated on different size plates and allowed to grow to the desired degree of confluence. The confluent cells were then treated with 50, 100, and $150 \mu \mathrm{g} / \mathrm{mL}$ AGEs for 24 hours. To test the effect of loratadine, 25 and $50 \mu \mathrm{M}$ concentrations of the drug were added to the cell media during the same time window as the AGEs. SW1353 cells were purchased from the American Type Culture Collection (Manassas, USA). Experiments were approved by the ethics committee of Jilin University.

\section{RNA Purification and Real-Time PCR}

The total RNAs from SW1353 cells were extracted using a commercial High Pure RNA Kit (Roche, USA). The purification was based on the mini-column absorption and elution method, and all the steps were performed by following the manufacturer's instructions. The quality and concentration of the isolated RNAs were measured by Nanodrop. To synthesize cDNA from RNA, $1 \mu \mathrm{g}$ of RNA from each sample was reacted with the template using an RT-PCR One Step Kit. For real-time PCR, SYBR Green PCR Master Mix was combined with the following human primers to monitor changes in their expression: H1R, NOX-4, TxNIP, NRF2, and GAPDH (as an internal control). The relative mRNA expression levels were normalized to that of GAPDH and calculated as fold-changes using the $2-\Delta \Delta \mathrm{CT}$ method.

\section{Western Blot Analysis}

Whole cell lysates of SW1353 were lysed with RIPA buffer plus protease and phosphatase inhibitors. After a short spin at maximum speed on a tabletop centrifuge, the supernatants 
were collected, and the concentration of soluble proteins was determined using a BCA Protein Quantitation Kit. A $20 \mu \mathrm{g}$ protein sample was immobilized on a $4-12 \%$ PAGE gel. The protein immobilized gel was then transferred to a PVDF membrane and blocked with 5\% nonfat milk in PBS, followed by incubation with the primary antibodies and a corresponding secondary antibody. Finally, the images of the blots were processed on an Odyssey automatic Western blotting system, and densitometric analysis was performed using the publicly available software Image J (NIH).

\section{Mitochondrial ROS Measurement}

The mitochondrial ROS level was measured by staining living SW1353 cells with $5 \mu \mathrm{M}$ MitoSOX Red (Life Science). Multiple images were taken at the same time with the same parameter settings using a Leica confocal microscope. The images were analyzed with the NIH software Image J. Data are presented as the percentage change from the control.

\section{ELISA}

The cell culture media was collected by centrifuging to remove cell debris. The production of IL-1 $\beta$ and IL-18 in the collected media was analyzed using cytokine-specific ELISA kits (R\&D Systems). The cytokine levels were extrapolated from the standard curve and normalized to the total media protein concentration.

\section{NRF2 Knockdown}

NRF2 expression was silenced by transfecting its specific siRNA or scramble oligos into SW1353 cells using Lipofectamine RNAi Max (Life Technology, USA) reagent. The transfected cells were then allowed to grow for an additional 48-72 hours to reach the silenced phase. The efficiency of NRF2 knockdown was assessed by Western blot analysis using NRF2specific antibody as described in 2.3 Western blot analysis.

\section{Statistics}

All the experiments performed in this study were repeated at least three times, and the data are presented as means \pm standard derivation (S.D.). Two-group comparisons were analyzed using a two-tailed non-paired Student's $t$-test. Multiple-group comparisons were tested using the one-way ANOVA method. The significance differences was determined based on a threshold of $\mathrm{p}<0.05$.

\section{Results}

\section{HIR Is Induced in Response to AGE Stimulation}

Firstly, we tested the response of H1R to AGE treatment in cultured SW1353 chondrocytes. Following treatment with a series of doses of AGEs (50, 100, and $150 \mu \mathrm{g} / \mathrm{mL}$ ) for 24 hours, we found that both the mRNA (Figure 1A) and protein (Figure 1B) expression of $\mathrm{H} 1 \mathrm{R}$ were induced in a dose-dependent manner.

\section{HIR Antagonist Loratadine Reduces AGE-Induced Increased Mitochondrial Reactive Oxygen Species (ROS)}

Next, we examined the effects of loratadine on the production of mitochondrial reactive oxygen species
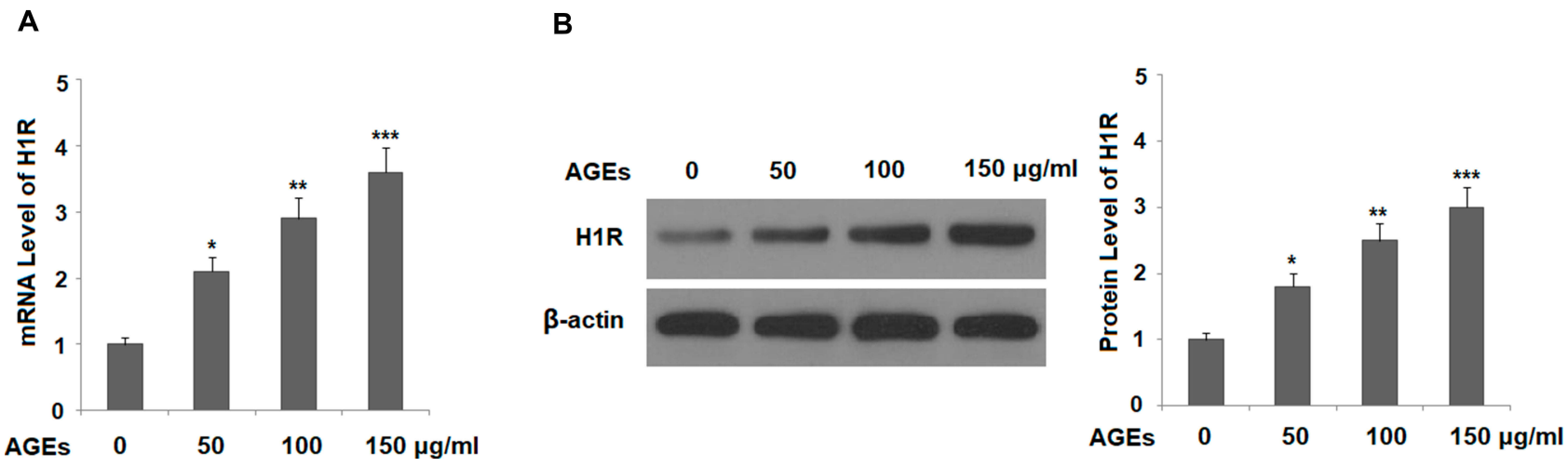

Figure I Advanced glycation end products (AGEs) increased the expression of the histamine HI type receptor (HIR) in human SWI353 chondrocytes. Cells were stimulated with AGEs at 50, I00, and $150 \mu \mathrm{g} / \mathrm{mL}$ for $24 \mathrm{~h}$. (A) mRNA of HIR; (B) Protein of HIR ( $* \mathrm{P}<0.0 \mathrm{I}$ vs vehicle group; $* * \mathrm{P}<0.00 \mathrm{I}$ vs vehicle group; $* * * \mathrm{P}<0.000 \mathrm{I}$ vs vehicle group). 


\section{AGEs}

Loratadine
0

0
100

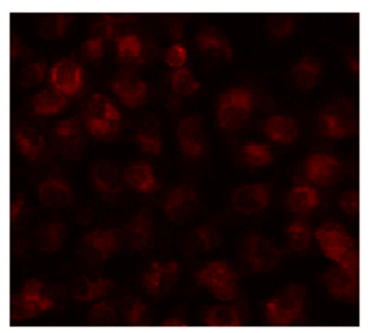

0

100

25

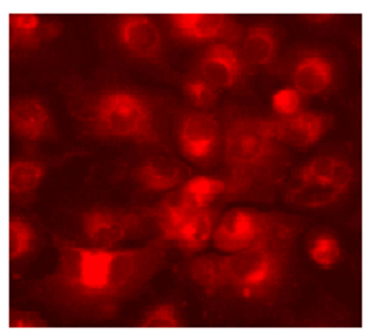

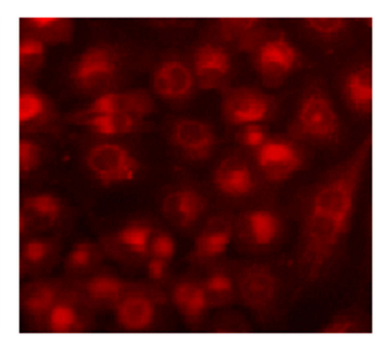

$100 \mu \mathrm{g} / \mathrm{ml}$

$50 \mu \mathrm{M}$
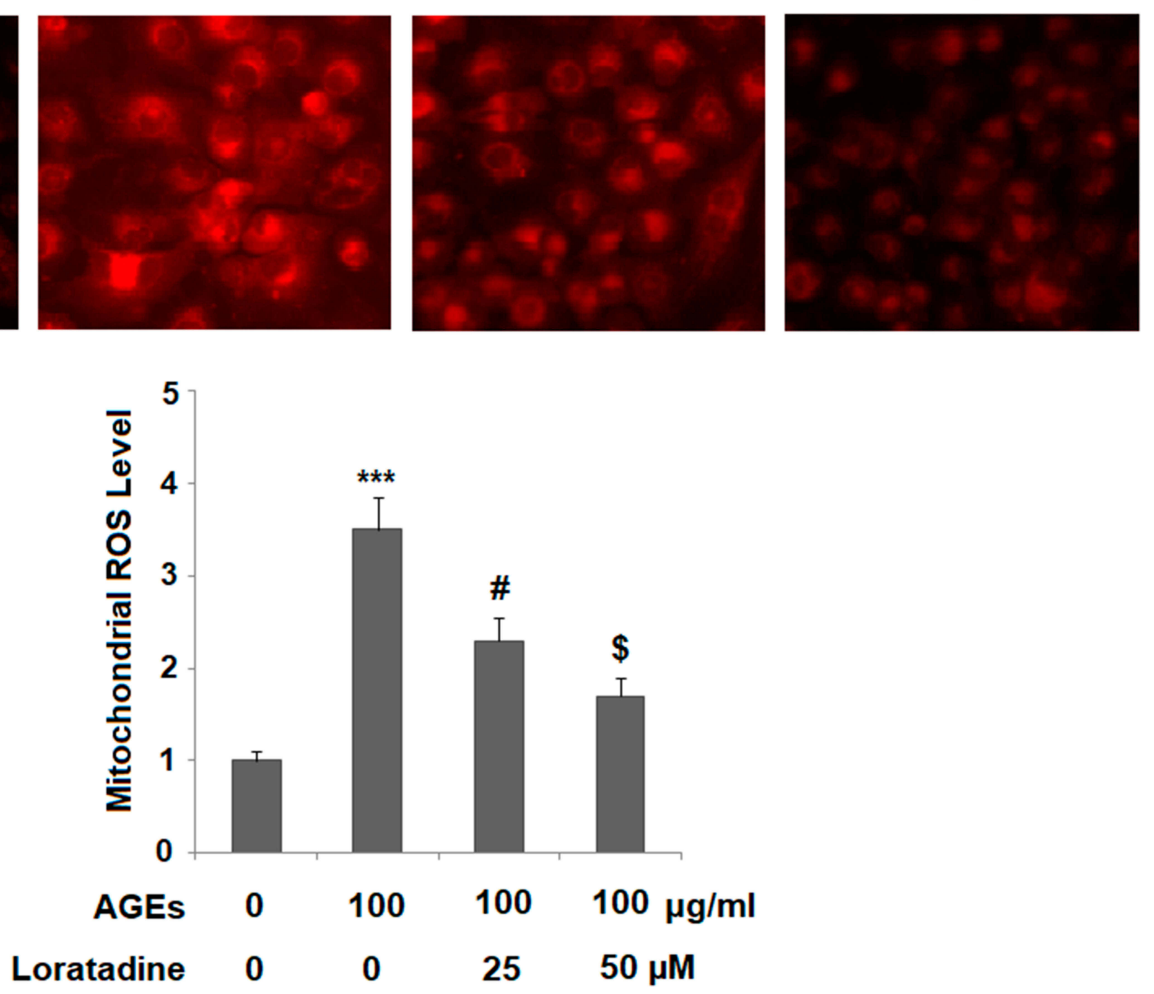

Figure 2 Treatment with loratadine reduced AGE-induced increased mitochondrial reactive oxygen species (ROS) in human SWI353 chondrocytes. Cells were stimulated with AGEs $(100 \mu \mathrm{g} / \mathrm{mL})$ in the presence or absence of loratadine $(25,50 \mu \mathrm{M})$ for $24 \mathrm{~h}$. Mitochondrial ROS was measured by the MITOSOX red staining $(* * * \mathrm{P}<0.000 \mathrm{I}$ vs vehicle group; ${ }^{\#} \mathrm{P}<0.01$ vs $\mathrm{AGEs}$ group; ${ }^{\$} \mathrm{P}<0.01$ vs $\mathrm{AGEs}+25 \mu \mathrm{M}$ loratadine).

(ROS). Our results indicate that AGEs induced severalfold ROS production, but the addition of the two doses of loratadine $(25,50 \mu \mathrm{M})$ dose-responsively suppressed the production of mitochondrial ROS (Figure 2).

\section{Loratadine Inhibits AGE-Induced Expression of NOX4}

To elucidate the effect of loratadine on ROS production, we assessed its influence on NADPH oxidase 4 (NOX4) in SW1353 cells. Consistently, we found that loratadine dose-responsively suppressed AGE-induced NOX4 expression at both the mRNA (Figure 3A) and protein levels (Figure 3B).

\section{Loratadine Inhibits AGE-Induced Expression of TxNIP}

We then assessed the influence of loratadine on the expression of the pro-inflammatory molecule TxNIP. Again, our results showed that loratadine dose-responsively suppressed AGE-induced TxNIP expression at both the mRNA (Figure 4A) and protein levels (Figure 4B).

\section{Loratadine Suppresses NLRP3 Inflammasome Activation}

To explore the mechanism of loratadine in chondrocytes, we examined the TxNIP/NLRP3 inflammasome complex in the presence or absence of the drug. Our experiments showed that the presence of loratadine dose-responsively suppressed AGE-induced expression of three key components of the NLRP3 inflammasome, including NLRP3, ASC, and cleaved caspase 1 (P10) (Figure 5), indicating that loratadine mitigates AGE-induced NLRP3 inflammasome activation.

\section{Loratadine Inhibits AGE-Induced Production of IL-I $\beta$ and IL- I 8}

We then assessed the effect of loratadine on the production of IL-1 $\beta$ and IL-18, two cytokines produced by NLRP3 inflammasome activation. The results of ELISA assay 
A

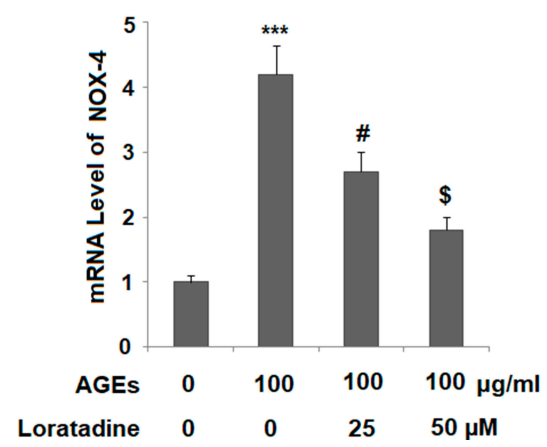

B

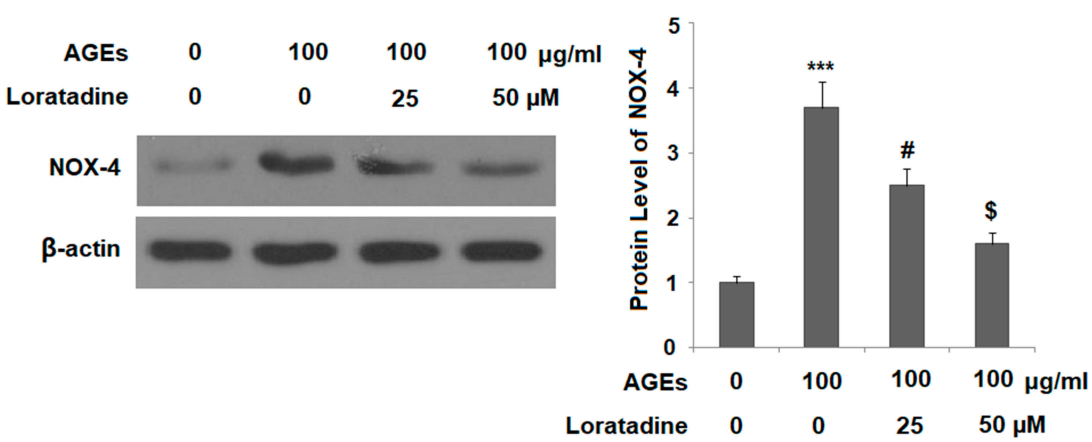

Figure 3 Treatment with loratadine reduced AGE-induced expression of NADPH oxidase 4 (NOX4) in human SWI353 chondrocytes. Cells were stimulated with AGEs $(100 \mu \mathrm{g} / \mathrm{mL})$ in the presence or absence of loratadine $(25,50 \mu \mathrm{M})$ for $24 \mathrm{~h}$. (A) mRNA of NOX4; (B) Protein expression of NOX-4 $\left(* * * \mathrm{P}<0.000 \mathrm{I}\right.$ vs vehicle group; ${ }^{\#} \mathrm{P}<0.0 \mathrm{I}$ vs AGEs group; ${ }^{\$} \mathrm{P}<0.01$ vs AGEs+25 $\mu$ M loratadine).

A

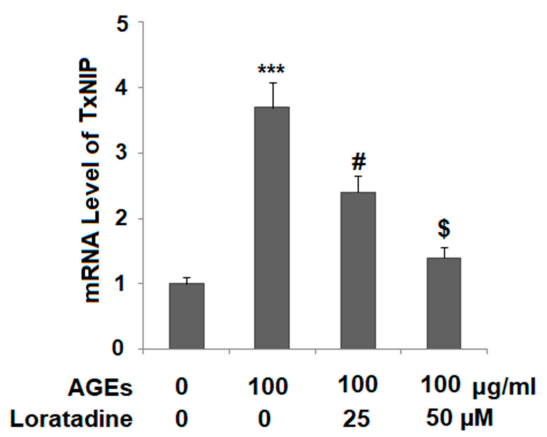

B

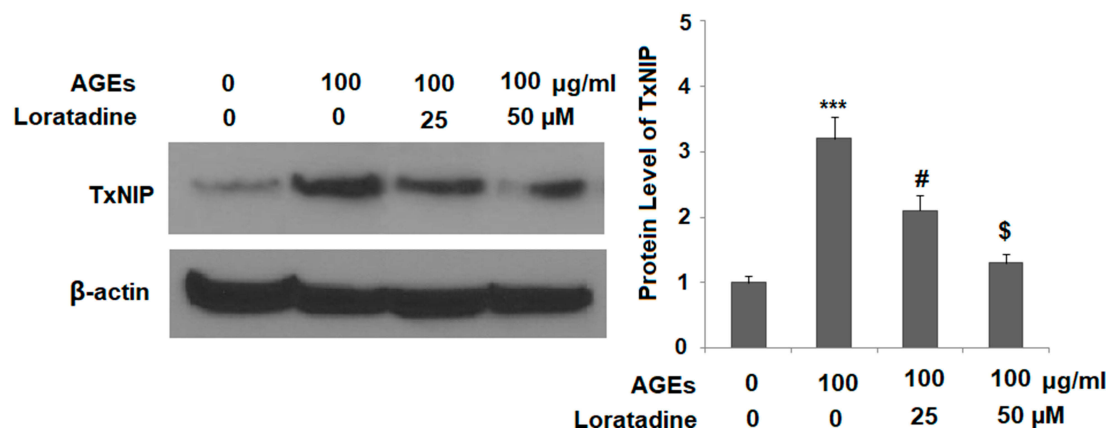

Figure 4 Treatment with the HIR antagonist loratadine decreased AGE-induced expression of TxNIP in human SWI353 chondrocytes. Cells were stimulated with AGEs $(100 \mu \mathrm{g} / \mathrm{mL})$ in the presence or absence of loratadine $(25,50 \mu \mathrm{M})$ for $24 \mathrm{~h}$. (A) mRNA of TxNIP; (B) Protein of TxNIP as measured by Western blot analysis (***P $<0.000 \mathrm{I}$ vs vehicle group; ${ }^{\#} \mathrm{P}<0.01$ vs $A G E s$ group; ${ }^{\$} \mathrm{P}<0.01$ vs $A G E s+25 \mu \mathrm{M}$ loratadine).

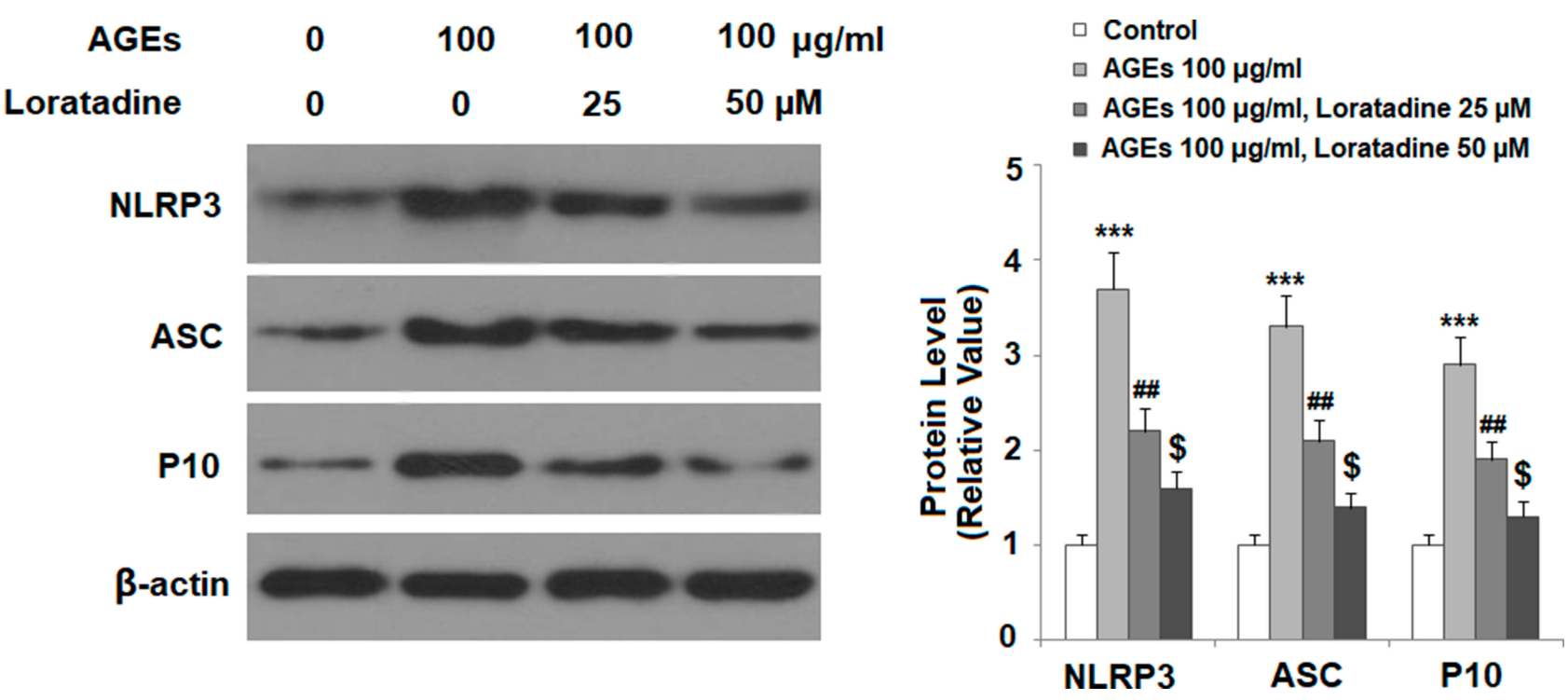

Figure 5 Treatment with the HIR antagonist loratadine prevented AGE-induced activation of the NLRP3 inflammasome in human SWI353 chondrocytes. Cells were stimulated with AGEs $(100 \mu \mathrm{g} / \mathrm{mL})$ in the presence or absence of loratadine $(25,50 \mu \mathrm{M})$ for $24 \mathrm{~h}$. Expression of NLRP3, ASC, and cleaved caspase I (PI0) was measured by Western blot analysis (***P< $<0.000$ I vs vehicle group; ${ }^{\#} \mathrm{P}<0.001$ vs $A G E s$ group; ${ }^{\$} \mathrm{P}<0.01$ vs $A G E s+25 \mu M$ loratadine). 
indicated that loratadine dose-responsively inhibited AGEinduced production of both IL-1 $\beta$ (Figure $6 \mathrm{~A}$ ) and IL-18 (Figure 6B).

\section{Loratadine Mitigates AGE-Mediated Reduced NRF2}

We also assessed the effect of loratadine on the expression of the antioxidant regulator NRF2. Treatment with AGEs reduced NRF2 expression, but the presence of the two doses of loratadine mitigated the AGE-induced decrease in NRF2, with the dose of $50 \mu \mathrm{M}$ nearly restoring both NRF2 mRNA (Figure 7A) and protein expression (Figure 7B).

\section{Silencing of NRF2 Abolishes the Inhibitory Effect of Loratadine on NLRP3 Inflammasome Activation}

Finally, we examined the effect of loratadine on the NLRP3 inflammasome when NRF2 was silenced in SW1353 cells. Our experiment verified that we silenced the protein expression of NRF 2 by nearly $60 \%$ (Figure $8 \mathrm{~A}$ ). Interestingly, loratadine lost its inhibitory action on AGE-induced NLRP3 inflammasome activation in NRF2-silent cells but not in NRF2-expressing cells (Figure 8B). Furthermore, the results of ELISA assay revealed that loratadine failed to show any effect on AGE-induced IL-18 production in
A

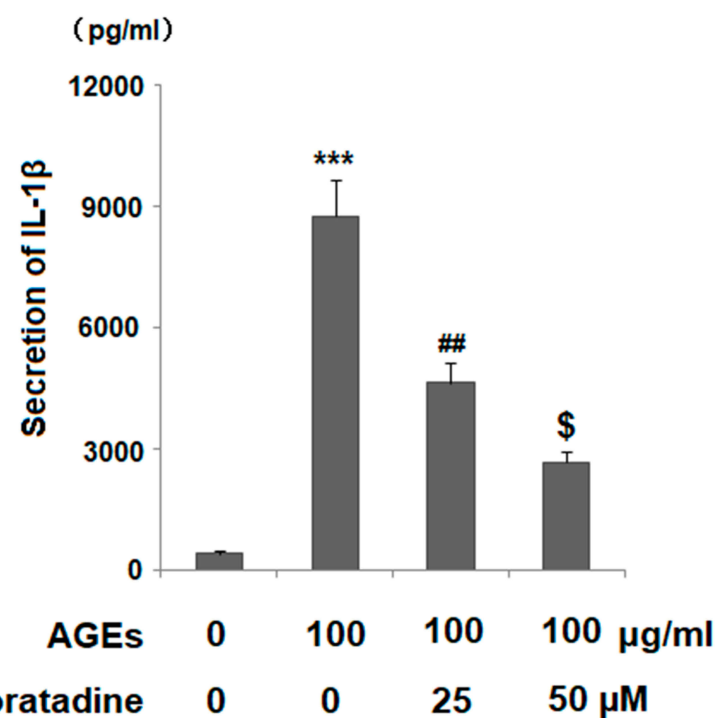

B

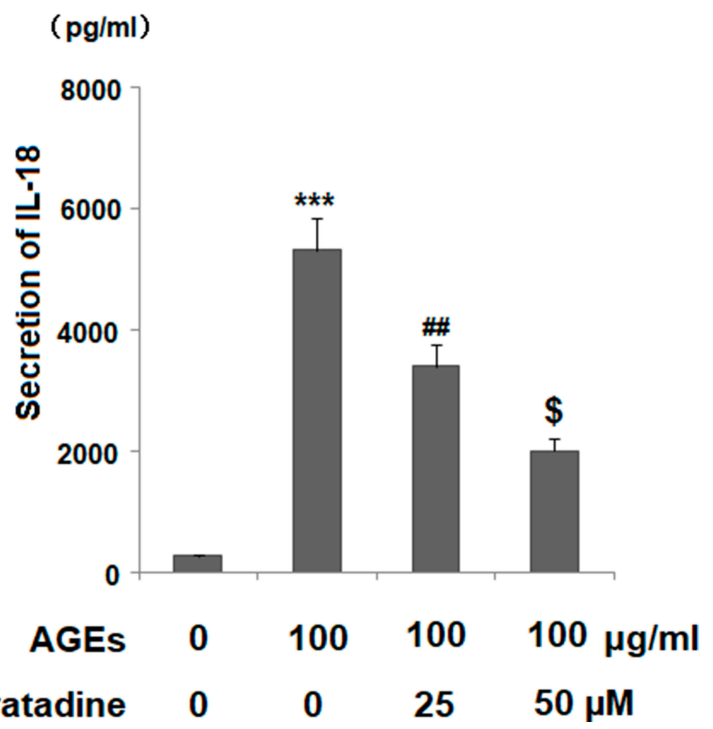

Figure 6 Treatment with the HIR antagonist loratadine prevented AGE-induced secretion of IL-I $\beta$ and IL-I8 in human SWI353 chondrocytes. Cells were stimulated with AGEs $(100 \mu \mathrm{g} / \mathrm{mL})$ in the presence or absence of loratadine $(25,50 \mu \mathrm{M})$ for $24 \mathrm{~h}$. (A) Secretion of IL- I $\beta$; (B) Secretion of IL- I8 $\left(* * * P<0.000\right.$ I vs vehicle group; ${ }^{\# \# P<0.00 I ~ v s ~}$ AGEs group; ${ }^{\$} \mathrm{P}<0.0 \mathrm{I}$ vs AGEs $+25 \mu \mathrm{M}$ loratadine).

A

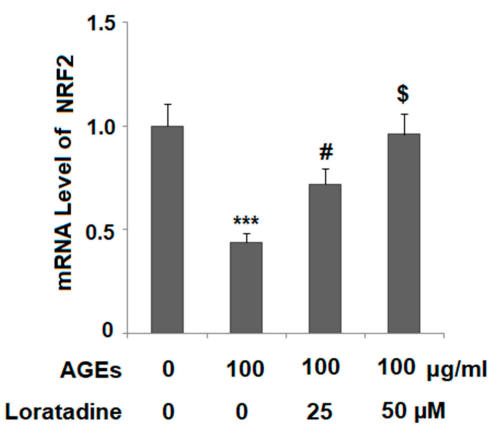

B

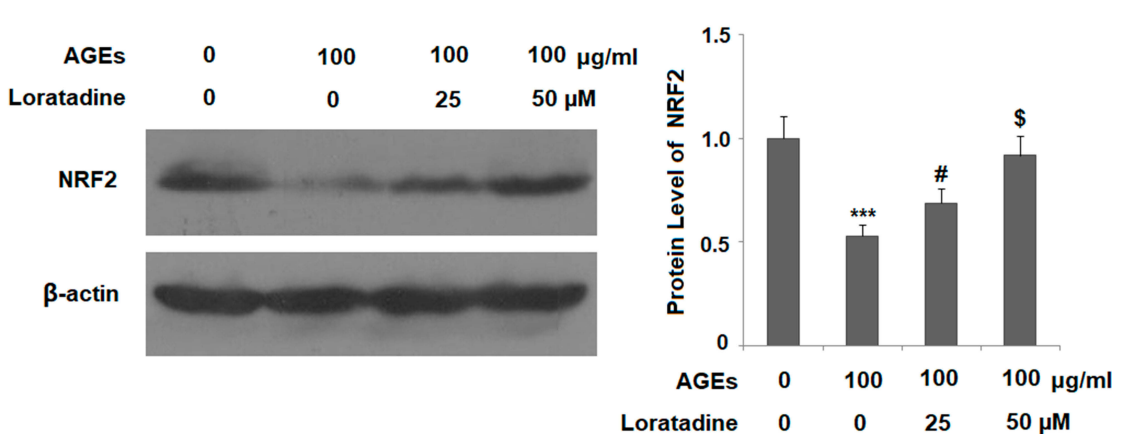

Figure 7 Treatment with the HIR antagonist loratadine restored AGE-induced reduced nuclear factor erythroid 2-related factor 2 (NRF2) expression in human SWI353 chondrocytes. Cells were stimulated with AGEs $(100 \mu \mathrm{g} / \mathrm{mL})$ in the presence or absence of loratadine $(25,50 \mu M)$ for 24 h. (A) mRNA of NRF2; (B) Protein of NRF2 (*** $\mathrm{P}<0.0001$ vs vehicle group; ${ }^{*} \mathrm{P}<0.01$ vs $A G E s$ group; ${ }^{\$} \mathrm{P}<0.01$ vs $A G E s+25 \mu M$ loratadine). 
A
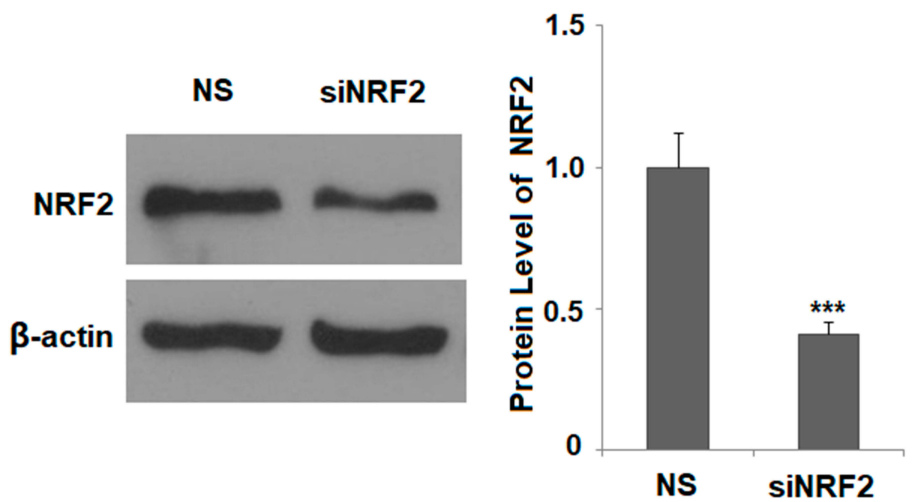

C

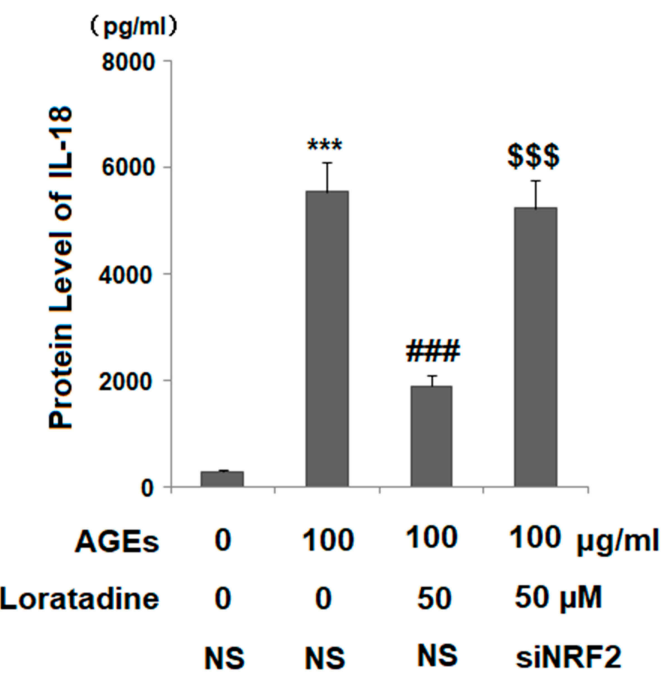

B

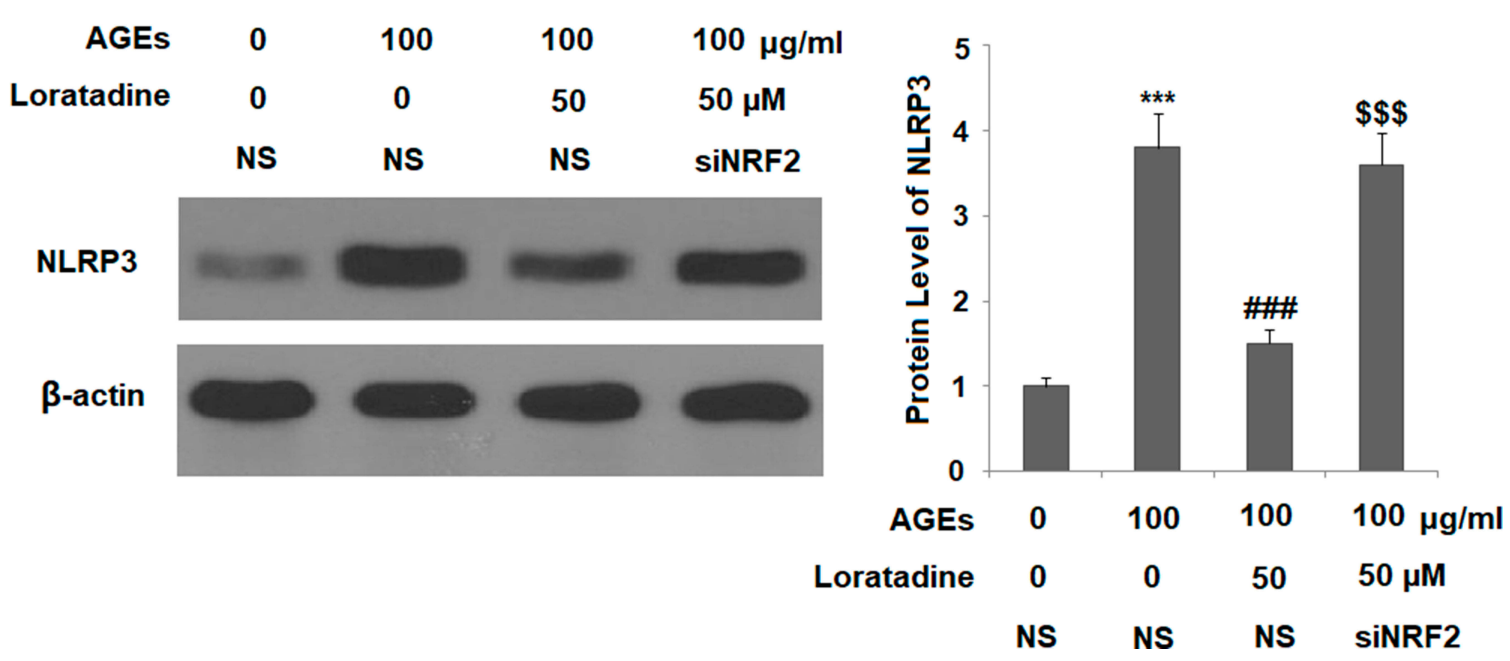

Figure 8 Knockdown of NRF2 abolished the protective effects of loratadine against NLRP3 activation in human SWI 353 chondrocytes. Cells were transfected with NRF2 siRNA. At $24 \mathrm{~h}$ post transfection, cells were stimulated with AGEs $(100 \mu \mathrm{g} / \mathrm{mL})$ in the presence or absence of loratadine $(50 \mu \mathrm{M})$ for $24 \mathrm{~h}$. (A) Western blot revealed the successful knockdown of NRF2; NS, non-specific siRNA; siNRF2, NRF2 siRNA; (B) Protein expression of NLRP3 as measured by Western blot analysis; (C) Secretion of IL18 as measured by ELISA ( ${ }^{* * *} \mathrm{P}<0.000$ I vs NS group; ${ }^{m} \mathrm{P}<0.000$ I vs AGEs +NS group; ${ }^{\$ \$} \mathrm{P}<0.000 \mathrm{I}$ vs $\mathrm{AGEs}+50 \mu \mathrm{M}$ loratadine).

NRF2-silent cells but maintained its inhibitory effect in NRF2-expressing cells (Figure $8 \mathrm{C}$ ). These data suggest that the expression of NRF2 in chondrocytes is necessary for the action of loratadine in the context of AGE stimulation.

\section{Discussion}

Histamine acts by binding to $\mathrm{G}$ protein-coupled histamine receptors, including $\mathrm{H} 1, \mathrm{H} 2, \mathrm{H} 3$, and $\mathrm{H} 4$, which have differing degrees of affinity to histamine. Among them, the two most sensitive receptors are the $\mathrm{H} 3$ and $\mathrm{H} 4$ receptors, while the activation of $\mathrm{H} 1$ and $\mathrm{H} 2$ receptors requires significantly higher concentrations of histamine. ${ }^{18}$ The activation of the
H1 receptor in chondrocytes is associated with the synthesis of the extracellular matrix. ${ }^{12}$ A recent study indicated that residential mast cells in synovial tissue also play an important role in OA and contribute to the structural damage of cartilage in OA. ${ }^{19}$ Epidemiological population-based studies have found that the use of H1R type antihistamine drugs is associated with a reduced prevalence of knee $\mathrm{OA},{ }^{20}$ indicating the possible role of loratadine in OA.

In recent years, targeted modulation of chondrocyte function has been implicated in the treatment of osteoarthritis and other joint diseases. During the development of osteoarthritis, chondrocyte renewal fails to satisfy the need 
for the repair of damaged cartilage tissue. Targeted drug intervention in chondrocytes offers the potential to recover destructive chondrocytes to their normal function. ${ }^{21}$ Previous work has shown that the histamine receptor $\mathrm{H} 1 \mathrm{R}$ is detectable in osteoarthritic cartilage, ${ }^{22}$ and the stimulation of H1R by histamine induces chondrocyte proliferation, ${ }^{11}$ suggesting that H1R is a feasible treatment target in chondrocytes. Chondrocytes synthesize large amounts of proteoglycans, which is an important component of the cartilage matrix. Research has shown that the stimulation of H1R increases proteoglycan synthesis. ${ }^{12}$ All these findings indicate that targeting H1R in chondrocytes to reverse chondrocyte dysfunction has the potential to treat osteoarthritis and cartilage damage. However, the regulatory mechanism of H1R in chondrocytes remains to be investigated. AGEs are known to be an important stimulator of chondrocyte activation, and its damaging effect on chondrocytes has been well documented. ${ }^{23}$

In our study, we used AGEs to induce chondrocyte activation and showed that loratadine had a protective effect against AGE-induced chondrocyte damage. We initiated our investigation and showed that H1R was induced in response to AGE treatment in a dosedependent manner in SW1353 cells, suggesting that stressinduced H1R expression could play a role in chondrocyte activation. We then assessed the impact of the loss of this receptor on chondrocyte activation.

Our results show that loratadine treatment exerted a protective effect against AGE-induced chondrocyte activation. Of note, loratadine treatment exerted an ameliorative effect on AGE-induced ROS production, as revealed by decreased mitochondrial ROS production and cytosol NOX4 expression. Furthermore, treatment with loratadine suppressed the AGE-induced expression of proinflammatory TxNIP. TxNIP is a vital mediator of ROS signaling, and previous work has shown that ROS serve as danger signals to link oxidative stress and inflammasome activation. ${ }^{24}$ Next, we fully examined the impact of loratadine on NLRP3 inflammasome activation. The activation of the NLRP3 inflammasome complex facilitates the production of IL- $1 \beta$ and IL-18 involving the adaptor protein ASC and cleavage of caspase $1 .{ }^{25}$ Our data also confirm that loratadine suppressed the AGE-induced expression of ASC and the cleavage product of caspase 1 , thereby reducing the production of IL-1 $\beta$ and IL-18 by the NLRP 3 inflammasome. Moreover, we showed that loratadine inhibited the expression of the anti-inflammatory regulator NRF2. NRF2 is a critical negative regulator of NLRP3 inflammasome activation. ${ }^{26}$ When NRF2 was silenced in chondrocytes, loratadine lost all of its inhibitory effects on NLRP3 inflammasome activation, suggesting that chondrocyte NRF2 is an upstream signal of the NLRP3 inflammasome regulated by H1R. The abnormally high expression of NRF2 has been linked to cartilage damage and has been recognized as a promising molecular target in the treatment of osteoarthritis. ${ }^{27} \mathrm{~A}$ recent drug repository study shows that a different H1R antagonist Astemizole promotes the expression of several target genes regulated by NRF2, indicating that NRF2 could be in the regulatory scope of H1R signaling. ${ }^{28}$ In lieu of this study, we showed direct evidence that histamine receptor signals act by increasing NRF2 expression to counter against AGE-induced cellular stress in chondrocytes. The remaining question is how H1R blockade influences NRF2 expression. Previous work has shown that NRF2 shuttles between cytosol and the nucleus via multiple signaling pathways. ${ }^{29}$ As a transcriptional factor, NRF2 regulates cellular ROS and inflammation mainly through transcription of its target genes in the nucleus. We hypothesize that loratadine may promote nuclear entry of NRF2 where it controls the transcription of the NLRP3 inflammasome complex.

Usually, loratadine acts as an antagonist of H1R, which is activated by histamine. In the current study, our results indicate that loratadine alone exhibits a protective role against AGEsinduced cellular stress in chondrocytes, suggesting that loratadine could have a direct impact on chondrocytes other than through H1R-dependent signaling. Previous studies have made a similar observation that loratadine treatment could regulate the function of vascular endothelial cells, bone marrow derived dendritic cells and eosinophils through a mechanism independent of H1R antagonism. ${ }^{30-32}$ Thus, we conclude that loratadine might regulate alternative pathways in chondrocytes, which might occur independently of H1R. Further investigations in the future will provide a complete picture of the underlying mechanism.

There are a few limitations to our study. Firstly, all the tests were performed in SW1353 cells, which are derived from chondrosarcoma cells. Although SW1353 was considered as a surrogate cell line for primary chondrocytes, it shows limited potential to mimic primary chondrocytes. ${ }^{33}$ Ideally, tests using primary isolated chondrocytes from OA patients would provide validation of the effect of loratadine. Also, immortalized chondrocytes cultured under conventional conditions tend to undergo dedifferentiation that features a loss of the chondrocyte phenotype and adoption of the fibroblast phenotype. ${ }^{34}$ Secondly, the pathogenesis of OA is 
a complex process involving multiple cell types and their interactions. Although chondrocytes play a significant role in OA development, other cell types, such as synoviocytes and immune cells, contribute as well. Therefore, the ultimate test of the therapeutic effect of loratadine using in vivo studies with animal models is necessary in the future.

In conclusion, our study demonstrates that loratadine mitigates AGE-induced cellular stress in chondrocytes. Loratadine is a widely used generic drug for relief of the allergic response, and its protective effect in chondrocytes is worthy of our attention for further investigation. It is conceivable that loratadine could have the potential to be used in the treatment of degenerative osteoarthritis and cartilage injury.

\section{Ethical Statement}

SW1353 cells were purchased from the American Type Culture Collection (Manassas, USA). Experiments were approved by the ethics committee of Jilin University.

\section{Disclosure}

The authors report no conflicts of interest in this work.

\section{References}

1. Akkiraju H, Nohe A. Role of chondrocytes in cartilage formation, progression of osteoarthritis and cartilage regeneration. J Dev Biol. 2015;3(4):177-192. doi:10.3390/jdb3040177

2. Zuscik MJ, Hilton MJ, Zhang X, Chen D, O'Keefe RJ. Regulation of chondrogenesis and chondrocyte differentiation by stress. J Clin Invest. 2008;118(2):429-438. doi:10.1172/JCI34174

3. Byun K, Yoo Y, Son M, et al. Advanced glycation end-products produced systemically and by macrophages: A common contributor to inflammation and degenerative diseases. Pharmacol Ther 2017;177:44-55. doi:10.1016/j.pharmthera.2017.02.030

4. Saudek DM, Kay J. Advanced glycation endproducts and osteoarthritis. Curr Rheumatol Rep. 2003;5(1):33-40. doi:10.1007/ s11926-003-0081-x

5. Son S, Hwang I, Han SH, Shin JS, Shin OS, Yu JW. Advanced glycation end products impair NLRP3 inflammasome-mediated innate immune responses in macrophages. J Biol Chem. 2017;292 (50):20437-20448. doi:10.1074/jbc.M117.806307

6. Ahmed SM, Luo L, Namani A, Wang XJ, Tang X. Nrf2 signaling pathway: pivotal roles in inflammation. Biochim Biophys Acta Mol Basis Dis. 2017;1863(2):585-597. doi:10.1016/j.bbadis.2016.11.005

7. Jhang JJ, Yen GC. The role of Nrf2 in NLRP3 inflammasome activation. Cell Mol Immunol. 2017;14(12):1011-1012. doi:10.1038/ cmi.2017.114

8. White MV. The role of histamine in allergic diseases. J Allergy Clin Immunol. 1990;86(4 Pt 2):599-605. doi:10.1016/S0091-6749(05) 80223-4

9. Taylor DJ, Woolley DE. Evidence for both histamine H1 and H2 receptors on human articular chondrocytes. Ann Rheum Dis. 1987;46 (6):431-435. doi:10.1136/ard.46.6.431

10. Tetlow LC, Woolley DE. Histamine stimulates the proliferation of human articular chondrocytes in vitro and is expressed by chondrocytes in osteoarthritic cartilage. Ann Rheum Dis. 2003;62(10):9 91-994. doi:10.1136/ard.62.10.991
11. Tetlow LC, Woolley DE. Histamine stimulates matrix metalloproteinase-3 and -13 production by human articular chondrocytes in vitro. Ann Rheum Dis. 2002;61:737-740. doi:10.1136/ard.61.8.737

12. Sohen S, Ooe H, Hashima M, Nonaka T, Fukuda K, Hamanishi C. Activation of histamine $\mathrm{H} 1$ receptor results in enhanced proteoglycan synthesis by human articular chondrocyte: involvement of protein kinase $\mathrm{C}$ and intracellular $\mathrm{Ca}(2+)$. Pathophysiology. 2001;8 (2):93-98. doi:10.1016/S0928-4680(01)00066-9

13. May JR, Dolen WK. Management of allergic rhinitis: a review for the community pharmacist. Clin Ther. 2017;39(12):2410-2419. doi:10. 1016/j.clinthera.2017.10.006

14. Belsito DV. Second-generation antihistamines for the treatment of chronic idiopathic urticaria. J Drugs Dermatol. 2010;9(5):503-512.

15. Bachert C, Maspero J. Efficacy of second-generation antihistamines in patients with allergic rhinitis and comorbid asthma. $J$ Asthma. 2011;48(9):965-973. doi:10.3109/02770903.2011.616616

16. Raithel M, Nägel A, Zopf Y, et al. Plasma histamine levels $(\mathrm{H})$ during adjunctive H1-receptor antagonist treatment with loratadine in patients with active inflammatory bowel disease (IBD). Inflamm Res. 2010;59(Suppl 2):S257-8. doi:10.1007/s00011-009$0120-9$

17. Mondillo C, Varela ML, Abiuso AMB, Vázquez R. Potential negative effects of anti-histamines on male reproductive function. Reproduction. 2018;155(5):R221-R227. doi:10.1530/REP-17-0685

18. Tiligada E, Zampeli E, Sander K, Stark H. Histamine H3 and H4 receptors as novel drug targets. Expert Opin Investig Drugs. 2009;18:1519-1531. doi:10.1517/14728220903188438

19. de Lange-brokaar BJ, Kloppenburg $\mathrm{M}$, Andersen $\mathrm{SN}$, et al. Characterization of synovial mast cells in knee osteoarthritis: association with clinical parameters. Osteoarthritis Cartilage. 2016;24 (4):664-671. doi:10.1016/j.joca.2015.11.011

20. Shirinsky I, Shirinsky VH. (1)-antihistamines are associated with lower prevalence of radiographic knee osteoarthritis: a cross-sectional analysis of the osteoarthritis initiative data. Arthritis Res Ther. 2018;20(1):116. doi:10.1186/s13075-018-1619-7

21. Goldring MB. Update on the biology of the chondrocyte and new approaches to treating cartilage diseases. Best Pract Res Clin Rheumatol. 2006;20(5):1003-1025. doi:10.1016/j.berh.2006.06. 003

22. Tetlow LC, Woolley DE. Histamine, histamine receptors (H1 and $\mathrm{H} 2$ ), and histidine decarboxylase expression by chondrocytes of osteoarthritic cartilage: an immunohistochemical study. Rheumatol Int. 2005;26(2):173-178. doi:10.1007/s00296-005-0622-x

23. Steenvoorden MM, Huizinga TW, Verzijl N, et al. Activation of receptor for advanced glycation end products in osteoarthritis leads to increased stimulation of chondrocytes and synoviocytes. Arthritis Rheum. 2006;54(1):253-263. doi:10.1002/art.21523

24. Lane T, Flam B, Lockey R, Kolliputi N. TXNIP shuttling: missing link between oxidative stress and inflammasome activation. Front Physiol. 2013;4:50. doi:10.3389/fphys.2013.00050

25. Sutterwala FS, Haasken S, Cassel SL. Mechanism of NLRP3 inflammasome activation. Ann N Y Acad Sci. 2014;1319:82-95. doi:10.1111/nyas. 12458

26. Khan NM, Ahmad I, Haqqi TM. Nrf2/ARE pathway attenuates oxidative and apoptotic response in human osteoarthritis chondrocytes by activating ERK1/2/ELK1-P70S6K-P90RSK signaling axis. Free Radic Biol Med. 2018;116:159-171. doi:10.1016/j.freeradbiomed.2018.01. 013

27. Marchev AS, Dimitrova PA, Burns AJ, Kostov RV, DinkovaKostova AT, Georgiev MI. Oxidative stress and chronic inflammation in osteoarthritis: can NRF2 counteract these partners in crime? Ann N Y Acad Sci. 2017;1401(1):114-135. doi:10.1111/ nyas. 13407

28. Zhang QY, Chu XY, Jiang LH, Liu MY, Mei ZL, Zhang HY. Identification of non-electrophilic Nrf2 activators from approved drugs. Molecules. 2017;22:6. 
29. Theodore M, Kawai Y, Yang J, et al. Multiple nuclear localization signals function in the nuclear import of the transcription factor Nrf2. $J$ Biol Chem. 2008;283(14):8984-8994. doi:10.1074/jbc.M709040 200

30. Zhou Y, Gao C, Wang H, Liu L, Huang Z, Fa X. Histamine H1 type receptor antagonist loratadine ameliorates oxidized LDL induced endothelial dysfunction. Biomed Pharmacother. 2018;106:144 8-1453. doi:10.1016/j.biopha.2018.07.025

31. Wang M, Han J, Domenico J, Shin YS, Jia Y, Gelfand EW. Combined blockade of the histamine $\mathrm{H} 1$ and $\mathrm{H} 4$ receptor suppresses peanut-induced intestinal anaphylaxis by regulating dendritic cell function. Allergy. 2016;71(11):1561-1574. doi:10.1111/all.12904
32. Eda R, Sugiyama H, Hopp RJ, Bewtra AK, Townley RG. Effect of loratadine on human eosinophil function in vitro. Ann Allergy. 1993;71(4):373-378.

33. Gebauer M, Saas J, Sohler F, et al. Comparison of the chondrosarcoma cell line SW1353 with primary human adult articular chondrocytes with regard to their gene expression profile and reactivity to IL-1beta. Osteoarthritis Cartilage. 2005;13(8):697-708. doi:10.1016/ j.joca.2005.04.004

34. von der Mark K, Gauss V, von der Mark M, Muller P. Relationship between cell shape and type of collagen synthesised as chondrocytes lose their cartilage phenotype in culture. Nature. 1977;267 (5611):531-532. doi:10.1038/267531a0

\section{Publish your work in this journal}

Drug Design, Development and Therapy is an international, peerreviewed open-access journal that spans the spectrum of drug design and development through to clinical applications. Clinical outcomes, patient safety, and programs for the development and effective, safe, and sustained use of medicines are a feature of the journal, which has also been accepted for indexing on PubMed Central. The manuscript management system is completely online and includes a very quick and fair peer-review system, which is all easy to use. Visit http://www. dovepress.com/testimonials.php to read real quotes from published authors. 